\title{
Macrofaunal communities of threatened subtidal maërl seabeds on Tenerife (Canary Islands, north-east Atlantic Ocean) in summer
}

\author{
Rodrigo Riera ${ }^{1 *}$, Juan Domingo Delgado ${ }^{2}$, Myriam Rodríguez $^{1}$, Óscar Monterroso ${ }^{1}$, Eva Ramos $^{1}$ \\ ${ }^{1}$ Centro de Investigaciones Medioambientales del Atlántico (CIMA SL), C/Arzobispo Elías Yanes, \\ 44, 38206 La Laguna, Tenerife, Canary Islands, Spain \\ 2 Área de Ecología, Departamento de Sistemas Físicos, Químicos y Naturales. Facultad de Ciencias \\ Experimentales, Universidad Pablo de Olavide, Ctra. de Utrera km. 1, 41013 Sevilla, Spain
}

Received 9 October 2010; accepted 18 April 2011

(C)The Chinese Society of Oceanography and Springer-Verlag Berlin Heidelberg 2012

\begin{abstract}
This study contributes with the first data on physical and taxonomical structure of macrofaunal assemblages of maërl beds from the Canary Islands. Maërl beds and Cymodocea nodosa meadows of the Canary Islands are considered biodiversity hot-spots in terms of taxonomic and functional biodiversity with a broad geographical and depth ranges. The authors have studied the structure of the macrofaunal assemblages on different habitat types (Cymodocea, Caulerpa, sabellid field, garden eel and maërl beds). Samples were taken at a range of depths between 14 and $46 \mathrm{~m}$. Correlations were performed among abiotic variables (granulometry, organic matter, nitrogen and phosphates) and the most abundant taxa. Similarity analysis was performed to explore the patchiness of seabeds at a local scale. Significant differences were found in macrofaunal assemblages among seabed types, with highest abundances and lowest biodiversity in sabellid fields, where the sabellid Bispira viola dominated. The polychaetes Aponuphis bilineata and Chone filicauda and the mollusc Turritella brochii were the most abundant taxa on maërl beds. The mosaic of granulometric conditions would explain the associated macroinfaunal community structure and contribute to the creation of diversity on these relatively well preserved seabeds at a local scale.

Key words: Macrofauna, Polychaetes, Amphipods, Molluscs, soft-bottoms, subtidal, Tenerife, Canary Islands
\end{abstract}

\section{Introduction}

Maërl beds are, along with the Cymodocea nodosa meadows (a habitat type which is known locally as "sebadales"), threatened by several anthropogenic disturbances including dredging, eutrophication, fishing, harbour construction and mariculture (Ballesteros, 2006; Barberá et al., 2003). Loss of maërl habitat is exacerbated by its slow growth rate (Blake and Maggs, 2003). The conservation value of maërl seabeds in European waters is recognized under EU legislation (Habitats Directive 92/43/EEC, 1992, Annex V) and conventions (Convention for the protection of the Mediterranean Sea against pollution, 1976; Bern Convention, 1996; OSPAR convention, 1998) (Airoldi and Beck, 2007). Moreover, a special plan for the protection of Mediterranean coralligenous and maërl assemblages has been recently adopted within the frame- work of the United Nations Environment Programme's Mediterranean Action Plan (UNEP-MAP) (Agnesi et al., 2009), since these ecosystems have been recognized as habitats that support a particularly high biodiversity of flora and fauna (Sciberras et al., 2009).

Maërl beds cover large areas in the Canarian archipelago at depths of ca. $40 \mathrm{~m}$ to $150 \mathrm{~m}$ deep (pers. obs. and ecocartographic studies). However, there is no currently any plan or specific regional legislation related to their environmental protection in the Canary Islands, and as far as we know, no studies concerning basic questions of their actual community structure and conservation. Thus, for effective environmental monitoring assessment and management of maërl habitats in this archipelago, in-depth studies on the distribution, biodiversity and macrofaunal community structure were urgently required.

In this study, we have compared the macrofaunal

* Corresponding author, E-mail: rodrigo@cimacanarias.com 
community structure from different sedimentary habitats [Cymodocea meadows, Caulerpa meadows, Caulerpa and sabellids, maërl bottoms and seabeds colonized by garden eels, Heteroconger longissimus Günther (Pisces: Anguilliformes, Congridae)]. The study area may serve as a relatively well-preserved reference for this habitat mosaic. It is still characterized by the absence of periodical anthropogenic disturbances, although the installation of several finfish farms is envisaged for the near future. The aim of the present study is to characterize the macrobenthic assemblages of the subtidal seabed habitats on the south coast of the Tenerife Island, by examining composition, community structure and biodiversity of these sedimentary bottoms at a local scale, with a special interest in maërl bottoms of high conservation value.

\section{Material and methods}

The seabeds of Las Galletas, a fishing and touristic village on the south coast of Tenerife, are heterogeneous, with a mosaic of differentiated habitats occupying small patches. Eight stations were sampled in September 2005, selected to represent the five major sedimentary habitat types observed in the area: Maërl bottoms, Cymodocea meadows, Caulerpa meadows, Caulerpa and sabellids, and bottoms colonized by garden eels (Table 1, Fig. 1).

Three replicates were taken at each station, using

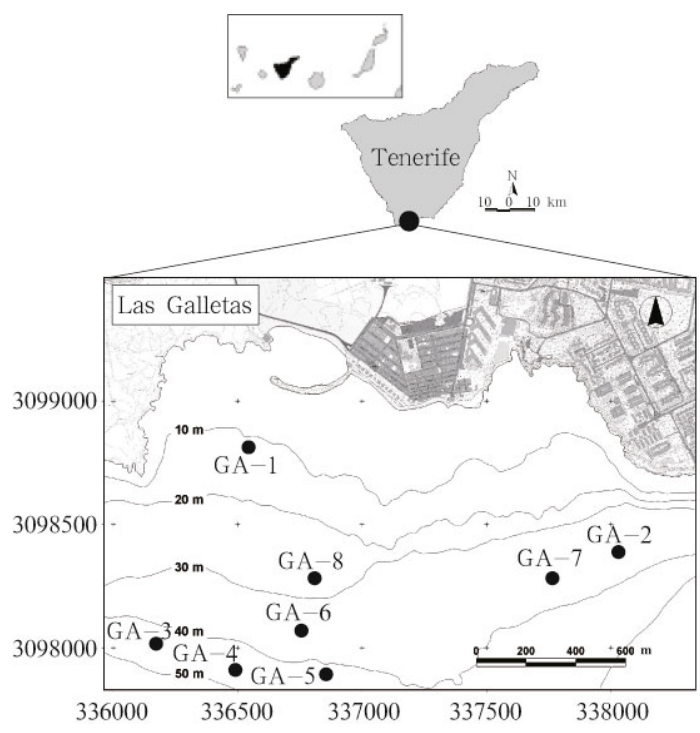

Fig.1. Map of the location of the Canarian archipelago (upper map), Tenerife Island and the study area at Las Galletas coast (South Tenerife) showing sampling stations (GA-1 to GA-8).
Table 1. Locations and depths of sampling stations

\begin{tabular}{ccccc}
\hline Station & UTM X & UTM Y & Depth/m & Community \\
\hline GA1 & 336396 & 3098838 & 14 & Cymodocea meadows \\
GA2 & 336230 & 3098275 & 38 & Caulerpa meadows \\
GA3 & 337335 & 3097611 & 45 & Maërl \\
GA4 & 337588 & 3097794 & 46 & Maërl \\
GA5 & 337821 & 3098026 & 46 & Maërl \\
GA6 & 338336 & 3098425 & 38 & Maërl \\
GA7 & 338084 & 3098401 & 39 & Caulerpa and \\
& & & & Sabellids \\
GA8 & 337979 & 3098590 & 29.8 & Garden eel \\
\hline
\end{tabular}

a $20 \mathrm{~cm}$ diameter core (area: $0.06 \mathrm{~m}^{2}$ ), inserted to a maximum depth of $20 \mathrm{~cm}$. Each sample was sieved through a $0.5 \mathrm{~mm}$ sieve, fixed in $4 \%$ formalin and latter transferred to $70 \%$ alcohol before sorting under a dissection microscope for macrofaunal identification. All macrofaunal specimens were determined to the lowest taxonomical level whenever possible.

Sedimentary samples were taken for abiotic factors (granulometry, organic matter, nitrogen and phosphates). These samples were taken by means of a 4.5 $\mathrm{cm}$ diameter core to a depth of $20 \mathrm{~cm}$. Granulometry samples were dried, sieved on a stack of graded sieves ranging from 63 to $2.000 \mu \mathrm{m}$ mesh, and the residue on each weighed (Buchanan and Kain, 1971). Organic carbon was determined by oxidizing carbon with $\mathrm{KMnO}_{4}$ (Walkley and Black, 1934). Total nitrogen content was determined by the Kjeldahl method (Kimberly and Roberts, 1905). Phosphates were determined by the direct UV measurement of the phosphomolybdate complex (Rubino et al., 1989).

Statistical analysis

Differences in the density of macrofauna between stations and habitats were examined using the nonparametric Kruskal-Wallis $(\mathrm{H})$ test. Spatial patterns in the macrofauna distribution were analysed using the PRIMER software package (Clarke and Warwick, 2001). To detect significant differences among macrofaunal assemblage composition (abundances, Shannon's diversity and Pielou's evenness) between habitats, multivariate analyses were carried out on untransformed and transformed (square root) abundance data. Correlations among the seven most abundant macrofaunal species and the relevant abiotic factors derived from the multivariate ordination were calculated with Spearman correlation coefficients. We have compared the mean values of the univariate descriptors (richness, abundance, evenness and diversity) among different bottom types by two-sided Student's t tests, adjusted for pairwise comparisons with the Bonf- 
erroni correction at $\alpha=0.05$. To find patterns of similarity among different sedimentary types in macrofaunal community structure, a hierarchical cluster analysis was performed with stations, using species composition and abundance per species. The Bray-Curtis similarity index with average linkage between groups was applied.

\section{Results and discussion}

\subsection{Abiotic variables}

Sedimentary fractions were in accordance with the sedimentary habitat variety and patchiness at a local scale (Fig. 2). Four sedimentary fractions characterized the sampling stations: fine sands in Cymodocea nodosa meadows (Sta. GA-1), medium sands in Caulerpa meadows (GA-2), Caulerpa and sabellids assemblages (GA-7) and garden eel bottoms (GA8). Mäerl seabeds were characterised by coarse sands (GA-3, GA-4 and GA-5) and medium-coarse sands (GA-6). Organic matter content and nitrogen concentration of the sediment presented low values in sampling stations (OM: $0.05 \%-0.25 \%$; N: $<1-3.0 \mathrm{mg} / \mathrm{kg}$ ). Phosphates showed low concentrations $(<20 \mathrm{mg} / \mathrm{kg})$, with the exception of Sta. GA-8 $(45.3 \mathrm{mg} / \mathrm{kg})$ (Table 2 ). However, this value could be considered to be intermediate in subtidal sandy seabeds of the Canarian archipelago (Riera R, pers. obs.).

\subsection{General faunal analysis}

A total of 1498 specimens belonging to 125 taxa

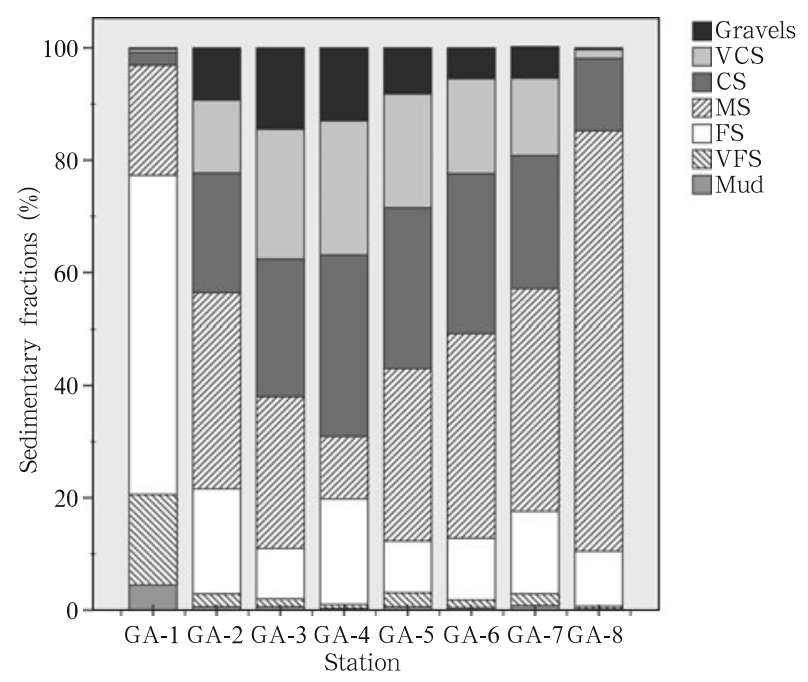

Fig.2. Sedimentary fractions of the sampling stations. VCS represents very coarse sands, CS coarse sands, MS medium sands, FS fine sands, and VFS very fine sands.
Table 2. Abiotic variables of the sampling stations (OM represents organic matter, $\mathrm{N}$ nitrogen, and $\mathrm{P}$ phosphates)

\begin{tabular}{cccc}
\hline Station & OM $(\%)$ & $\mathrm{N} / \mathrm{mg}^{\circ} \mathrm{kg}^{-1}$ & $\mathrm{P} / \mathrm{mg}^{-1 \mathrm{~kg}^{-1}}$ \\
\hline GA-1 & 0.14 & $<1$ & 19.6 \\
GA-2 & 0.10 & 2.4 & 14.7 \\
GA-3 & 0.16 & 2.2 & 12.7 \\
GA-4 & 0.20 & $<1$ & 6.8 \\
GA-5 & 0.21 & 3.0 & 10.8 \\
GA-6 & 0.05 & 2.4 & 13.3 \\
GA-7 & 0.15 & 2.8 & 15.6 \\
GA-8 & 0.25 & 2.2 & 45.3 \\
\hline
\end{tabular}

were collected. The most abundant taxonomic group was Polychaeta with 1109 specimens (74\% of the overall abundance). The remaining taxonomic groups were represented by less than 150 individuals. Cumaceans and mysids were extremely rare (1 and 2 specimens, respectively). The most diverse taxonomic groups were polychaetes (40 species) and decapods (21 species). Cumaceans, stomatopods, isopods, mysids and nemerteans were represented by one taxon (Table 3). The most abundant species was the polychaete sabellid Bispira viola with 509 individuals ( $\sim 34 \%$ of all individuals), followed by the polychaete onuphid Aponuphis bilineata with 258 individuals ( $\sim 17 \%$ of the total). The rest of species were represented by less than 70 specimens, with 43 species were represented by only one specimen (Table 3 ).

\subsection{Univariate indices}

Sampling stations were divided into five groups depending on habitat type: Cymodocea meadows, represented by Sta. GA-1; Caulerpa meadows (Sta. GA-2); Maërl (Stas GA-3, GA-4, GA-5 and GA-6); Caulerpa and sabellids (Sta. GA-7); Garden eels (Sta. GA-8).

The species richness ranged from ca. 11 species in Caulerpa meadows to 22 species in Caulerpa and sabellids. The differences in species richness among sedimentary habitats were not significant $(H=7.77$; $p=0.10$ ). The overall abundance varied greatly among sedimentary habitats, with maximum densities in the Caulerpa and sabellids with a mean of 204.33 specimens. The lowest densities were recorded in maërl bottoms with a mean of 35.8 individuals (Fig. 3). The differences in overall abundance among sedimentary habitats were significant $(H=11.04 ; p=0.02)$. The Shannon's diversity $\left(H^{\prime}\right)$ showed important variations among sedimentary habitats, with maximum values in garden eel bottoms and Cymodocea meadows with 2.47 and 2.45 , respectively. Contrarily, the lowest diversity values were recorded in Caulerpa and sabellid bottoms 
Table 3. Species list of the sampling stations

\begin{tabular}{|c|c|c|c|c|c|c|c|c|c|c|}
\hline Group & Species & GA-1 & GA-2 & GA-3 & GA-4 & GA-5 & GA-6 & GA-7 & GA-8 & Total \\
\hline Amphipoda & Amphilochus neapolitanus & 0 & 0 & 0 & 0 & 1 & 0 & 0 & 0 & 1 \\
\hline Amphipoda & Ampithoe rubricata & 1 & 0 & 0 & 0 & 0 & 1 & 0 & 0 & 2 \\
\hline Amphipoda & Corophium sp. & 1 & 0 & 0 & 0 & 0 & 0 & 0 & 1 & 2 \\
\hline Amphipoda & Corophium sp.1 & 0 & 0 & 0 & 0 & 0 & 1 & 0 & 0 & 1 \\
\hline Amphipoda & Dexamine spinosa & 0 & 0 & 0 & 0 & 0 & 0 & 1 & 0 & 1 \\
\hline Amphipoda & Gammaropsis maculata & 0 & 0 & 0 & 1 & 0 & 0 & 0 & 0 & 1 \\
\hline Amphipoda & Harpinia antennaria & 3 & 0 & 0 & 0 & 0 & 0 & 0 & 0 & 3 \\
\hline Amphipoda & Megaluropus agilis & 2 & 0 & 0 & 0 & 0 & 0 & 0 & 0 & 2 \\
\hline Amphipoda & Pariambus typicus & 4 & 0 & 0 & 0 & 0 & 0 & 0 & 0 & 4 \\
\hline Amphipoda & Photis longicaudata & 5 & 1 & 1 & 1 & 0 & 3 & 0 & 1 & 12 \\
\hline Amphipoda & Pseudoprotella phasma & 0 & 0 & 0 & 1 & 0 & 0 & 1 & 0 & 2 \\
\hline Amphipoda & Tryphosites longipes & 0 & 0 & 0 & 0 & 0 & 0 & 0 & 1 & 1 \\
\hline Amphipoda & Urothoe marina & 1 & 0 & 0 & 0 & 2 & 1 & 0 & 3 & 7 \\
\hline Amphipoda & Urothoe pulchella & 1 & 0 & 0 & 0 & 1 & 0 & 0 & 1 & 3 \\
\hline Cumacea & Iphinoe canariensis & 0 & 0 & 0 & 0 & 0 & 0 & 0 & 1 & 1 \\
\hline Decapoda & Alpheus dentipes & 0 & 0 & 0 & 4 & 0 & 0 & 0 & 0 & 4 \\
\hline Decapoda & Alpheus macrocheles & 0 & 0 & 0 & 2 & 0 & 0 & 7 & 0 & 9 \\
\hline Decapoda & Anapagurus laevis & 0 & 0 & 1 & 1 & 1 & 2 & 0 & 1 & 6 \\
\hline Decapoda & Atharas nitescens & 0 & 0 & 0 & 1 & 0 & 0 & 2 & 0 & 3 \\
\hline Decapoda & Calcinus tubularis & 0 & 0 & 1 & 3 & 0 & 0 & 3 & 0 & 7 \\
\hline Decapoda & Dardanus calidus & 0 & 0 & 0 & 0 & 0 & 0 & 2 & 0 & 2 \\
\hline Decapoda & Galathea intermedia & 0 & 0 & 1 & 5 & 0 & 0 & 5 & 0 & 11 \\
\hline Decapoda & Hippolyte longirostris & 0 & 0 & 0 & 0 & 0 & 0 & 1 & 0 & 1 \\
\hline Decapoda & Hippolyte varians & 0 & 0 & 1 & 0 & 0 & 0 & 2 & 0 & 3 \\
\hline Decapoda & Macropodia sp. & 0 & 0 & 0 & 0 & 0 & 0 & 1 & 0 & 1 \\
\hline Decapoda & Palaemus longirostris & 0 & 0 & 0 & 2 & 0 & 0 & 0 & 0 & 2 \\
\hline Decapoda & Palicus caronii & 0 & 0 & 0 & 0 & 0 & 0 & 1 & 0 & 1 \\
\hline Decapoda & Paractea monodi & 0 & 0 & 0 & 1 & 0 & 0 & 0 & 0 & 1 \\
\hline Decapoda & Parthenope massena & 0 & 0 & 1 & 0 & 0 & 1 & 0 & 0 & 2 \\
\hline Decapoda & Philocheras sculpus & 0 & 2 & 0 & 0 & 0 & 0 & 1 & 0 & 3 \\
\hline Decapoda & Philocheras trispinosus & 1 & 0 & 0 & 0 & 0 & 1 & 0 & 2 & 4 \\
\hline Decapoda & Pilumnus spirifes & 0 & 1 & 0 & 0 & 0 & 0 & 1 & 0 & 2 \\
\hline Decapoda & Pisa nodipes & 0 & 0 & 3 & 5 & 0 & 0 & 7 & 0 & 15 \\
\hline Decapoda & Polybius zariquieyi & 0 & 0 & 1 & 0 & 0 & 0 & 0 & 0 & 1 \\
\hline Decapoda & Processa canaliculata & 0 & 0 & 1 & 0 & 0 & 0 & 1 & 0 & 2 \\
\hline Decapoda & Nanocassiope melanodactyla & 0 & 0 & 0 & 0 & 0 & 0 & 3 & 0 & 3 \\
\hline Echinoidea & Ophiothrix fragilis & 1 & 0 & 0 & 1 & 0 & 3 & 0 & 0 & 5 \\
\hline Echinoidea & Ophiura texturata & 0 & 0 & 0 & 0 & 1 & 0 & 0 & 0 & 1 \\
\hline Echinoidea & Sphaerechinus granularis & 0 & 0 & 1 & 0 & 0 & 0 & 0 & 0 & 1 \\
\hline Echinoidea & Amplipholis squamata & 0 & 0 & 1 & 0 & 1 & 0 & 2 & 0 & 4 \\
\hline Echinoidea & Brissus unicolor & 3 & 0 & 0 & 0 & 0 & 0 & 0 & 1 & 4 \\
\hline Echinoidea & Echynociamus pusillus & 0 & 0 & 0 & 0 & 2 & 0 & 0 & 1 & 3 \\
\hline Holothuroidea & Holothuria fonskali & 0 & 0 & 0 & 1 & 0 & 0 & 0 & 0 & 1 \\
\hline Stomatopoda & Stomatopoda sp.1 & 0 & 0 & 0 & 0 & 0 & 1 & 2 & 0 & 3 \\
\hline Isopoda & Eurydice pulchra & 0 & 0 & 2 & 0 & 0 & 1 & 0 & 0 & 3 \\
\hline Misidacea & Gastrosaccus sanctus & 1 & 0 & 0 & 0 & 0 & 0 & 0 & 1 & 2 \\
\hline Nemertea & Nemertea sp.1 & 1 & 1 & 0 & 0 & 1 & 0 & 0 & 0 & 3 \\
\hline Ostracoda & Cypridina mediterranea & 2 & 0 & 0 & 0 & 0 & 0 & 1 & 0 & 3 \\
\hline Ostracoda & Cypridina norvergica & 2 & 0 & 0 & 0 & 0 & 0 & 0 & 2 & 4 \\
\hline Polychaeta & Aponuphis bilineata & 23 & 65 & 29 & 16 & 14 & 86 & 17 & 8 & 258 \\
\hline Polychaeta & Armandia polyophthalma & 0 & 1 & 0 & 0 & 0 & 0 & 0 & 0 & 1 \\
\hline Polychaeta & Aricidea assimilis & 0 & 0 & 1 & 0 & 0 & 0 & 1 & 0 & 2 \\
\hline Polychaeta & Armandia cirrosa & 0 & 0 & 0 & 0 & 0 & 0 & 0 & 3 & 3 \\
\hline Polychaeta & Bispira viola & 0 & 0 & 0 & 0 & 0 & 0 & 509 & 0 & 509 \\
\hline Polychaeta & Chone collaris & 0 & 0 & 0 & 3 & 0 & 1 & 3 & 0 & 7 \\
\hline Polychaeta & Chone filicaudata & 0 & 0 & 21 & 3 & 11 & 18 & 4 & 7 & 64 \\
\hline
\end{tabular}


Continued from Table 3

\begin{tabular}{|c|c|c|c|c|c|c|c|c|c|c|}
\hline Group & Species & GA-1 & GA-2 & GA-3 & GA-4 & GA-5 & GA-6 & GA-7 & GA-8 & Total \\
\hline Polychaeta & Chone sp. & 4 & 2 & 1 & 1 & 3 & 5 & 0 & 2 & 18 \\
\hline Polychaeta & Capitomastus minimus & 1 & 0 & 0 & 0 & 0 & 0 & 0 & 0 & 1 \\
\hline Polychaeta & Ditrupa arietina & 1 & 0 & 0 & 1 & 3 & 0 & 0 & 29 & 34 \\
\hline Polychaeta & Demonax brachychona & 0 & 58 & 0 & 0 & 0 & 1 & 0 & 0 & 59 \\
\hline Polychaeta & Glycera alba & 0 & 4 & 0 & 0 & 0 & 0 & 0 & 0 & 4 \\
\hline Polychaeta & Glycera sp. & 0 & 0 & 4 & 0 & 3 & 2 & 1 & 8 & 18 \\
\hline Polychaeta & Glycera sp.1 & 0 & 0 & 1 & 0 & 0 & 0 & 1 & 0 & 2 \\
\hline Polychaeta & Goniadides sp. & 0 & 0 & 0 & 1 & 0 & 1 & 0 & 0 & 2 \\
\hline Polychaeta & Harmothoe sp. & 0 & 0 & 0 & 0 & 0 & 0 & 1 & 0 & 1 \\
\hline Polychaeta & Harmothoe sp.1 & 0 & 0 & 1 & 2 & 0 & 1 & 2 & 0 & 6 \\
\hline Polychaeta & Hermione histryx & 0 & 0 & 0 & 2 & 0 & 0 & 0 & 0 & 2 \\
\hline Polychaeta & Hermodice carunculata & 0 & 0 & 0 & 4 & 0 & 1 & 5 & 0 & 10 \\
\hline Polychaeta & Hesione pantherina & 0 & 0 & 0 & 0 & 0 & 0 & 1 & 0 & 1 \\
\hline Polychaeta & Kefersteinia cirrata & 0 & 0 & 0 & 0 & 1 & 0 & 0 & 0 & 1 \\
\hline Polychaeta & Lumbrineriopsis paradoxa & 1 & 0 & 0 & 0 & 0 & 0 & 0 & 0 & 1 \\
\hline Polychaeta & Lumbrineris cingulata & 0 & 0 & 1 & 0 & 0 & 1 & 1 & 2 & 5 \\
\hline Polychaeta & Lumbrineris sp. & 0 & 2 & 0 & 0 & 0 & 0 & 0 & 0 & 2 \\
\hline Polychaeta & Malacoceros fuliginosus & 0 & 0 & 1 & 0 & 2 & 0 & 0 & 0 & 3 \\
\hline Polychaeta & Neanthes rubicunda & 0 & 0 & 3 & 3 & 0 & 0 & 1 & 0 & 7 \\
\hline Polychaeta & Nematonereis unicomis & 0 & 0 & 2 & 0 & 0 & 0 & 0 & 0 & 2 \\
\hline Polychaeta & Nephthys cirrosa & 4 & 0 & 0 & 0 & 0 & 0 & 0 & 7 & 11 \\
\hline Polychaeta & Petaloproctus terricola & 4 & 0 & 0 & 0 & 0 & 0 & 1 & 0 & 5 \\
\hline Polychaeta & Phyllodoce maculata & 0 & 1 & 0 & 0 & 0 & 0 & 0 & 0 & 1 \\
\hline Polychaeta & Phyllodoce sp. & 0 & 0 & 0 & 1 & 0 & 1 & 1 & 0 & 3 \\
\hline Polychaeta & Pista cristata & 0 & 1 & 0 & 0 & 0 & 0 & 0 & 0 & 1 \\
\hline Polychaeta & Poecilochaetus serpens & 0 & 1 & 1 & 1 & 0 & 2 & 0 & 3 & 8 \\
\hline Polychaeta & Prionospio steenstrupii & 0 & 10 & 0 & 2 & 2 & 0 & 0 & 25 & 39 \\
\hline Polychaeta & Rhynchospio glutaea & 0 & 1 & 0 & 0 & 0 & 0 & 0 & 0 & 1 \\
\hline Polychaeta & Scolelepis tridentata & 0 & 0 & 0 & 1 & 4 & 1 & 0 & 1 & 7 \\
\hline Polychaeta & Scoloplos (Leodamas) sp. & 0 & 3 & 1 & 0 & 0 & 0 & 6 & 1 & 11 \\
\hline Polychaeta & Scoloplos armiger & 0 & 0 & 1 & 1 & 0 & 2 & 2 & 4 & 10 \\
\hline Polychaeta & Sigalion squamatum & 15 & 1 & 3 & 2 & 4 & 1 & 0 & 4 & 30 \\
\hline Polychaeta & Syllis sp. & 0 & 0 & 0 & 1 & 0 & 0 & 0 & 0 & 1 \\
\hline Sipuncula & Aspidosiphon muelleri & 1 & 0 & 1 & 2 & 5 & 2 & 0 & 16 & 27 \\
\hline Sipuncula & Aspidosiphon sp. & 0 & 1 & 0 & 0 & 0 & 0 & 1 & 0 & 2 \\
\hline Sipuncula & Sipuncula sp. & 0 & 0 & 0 & 0 & 16 & 3 & 2 & 0 & 21 \\
\hline Sipuncula & Sipunculus nudus & 0 & 0 & 2 & 0 & 2 & 4 & 0 & 0 & 8 \\
\hline Tanaidacea & Apseudes talpa & 28 & 0 & 0 & 0 & 0 & 0 & 0 & 19 & 47 \\
\hline Tanaidacea & Leptochelia dubia & 2 & 0 & 1 & 0 & 0 & 1 & 2 & 0 & 6 \\
\hline Bivalvia & Callista chione & 0 & 0 & 0 & 0 & 0 & 0 & 1 & 1 & 2 \\
\hline Bivalvia & Chlamys corallinoides & 0 & 0 & 0 & 0 & 0 & 0 & 1 & 0 & 1 \\
\hline Bivalvia & Ervilia castanea & 0 & 0 & 1 & 0 & 0 & 0 & 0 & 0 & 1 \\
\hline Bivalvia & Gastrochaena dubia & 0 & 0 & 0 & 2 & 0 & 0 & 0 & 0 & 2 \\
\hline Bivalvia & Gregariella subclavata & 0 & 0 & 0 & 1 & 0 & 0 & 0 & 0 & 1 \\
\hline Bivalvia & Lima hians & 0 & 0 & 0 & 1 & 0 & 0 & 0 & 0 & 1 \\
\hline Bivalvia & Linga adansoni & 6 & 0 & 0 & 0 & 0 & 0 & 0 & 0 & 6 \\
\hline Bivalvia & Lucinella divaricata & 1 & 0 & 0 & 0 & 1 & 0 & 0 & 0 & 2 \\
\hline Bivalvia & Pandora pinna & 0 & 0 & 0 & 1 & 0 & 0 & 0 & 0 & 1 \\
\hline Bivalvia & Plagiocardium papillosum & 0 & 0 & 0 & 0 & 0 & 0 & 0 & 1 & 1 \\
\hline Bivalvia & Solemya togata & 5 & 0 & 0 & 0 & 0 & 1 & 1 & 0 & 7 \\
\hline Bivalvia & Tellina donacina & 0 & 0 & 0 & 0 & 0 & 1 & 0 & 0 & 1 \\
\hline Bivalvia & Thracia papyracea & 1 & 0 & 0 & 0 & 0 & 0 & 0 & 0 & 1 \\
\hline Gastropoda & Atys macandrewi & 0 & 0 & 1 & 0 & 0 & 0 & 0 & 0 & 1 \\
\hline Gastropoda & Bela ornata & 1 & 0 & 0 & 0 & 0 & 0 & 0 & 0 & 1 \\
\hline Gastropoda & Bittium incile & 0 & 0 & 0 & 0 & 0 & 1 & 0 & 0 & 1 \\
\hline Gastropoda & Bittium latreillii & 2 & 0 & 2 & 0 & 0 & 2 & 2 & 0 & 8 \\
\hline Gastropoda & Bulla mabillei & 0 & 0 & 1 & 2 & 1 & 0 & 0 & 0 & 4 \\
\hline
\end{tabular}


Continued from Table 3

\begin{tabular}{|c|c|c|c|c|c|c|c|c|c|c|}
\hline Group & Species & GA-1 & GA-2 & GA-3 & GA-4 & GA-5 & GA-6 & GA-7 & GA-8 & Total \\
\hline Gastropoda & Bursa marginata & 0 & 0 & 0 & 1 & 0 & 0 & 0 & 0 & 1 \\
\hline Gastropoda & Cylichna propecylindracea & 0 & 0 & 0 & 0 & 0 & 1 & 0 & 0 & 1 \\
\hline Gastropoda & Gibbula magus & 0 & 0 & 1 & 0 & 0 & 0 & 0 & 0 & 1 \\
\hline Gastropoda & Jujubinus exasperatus & 3 & 0 & 0 & 0 & 0 & 0 & 0 & 0 & 3 \\
\hline Gastropoda & Nassarius cuvierii & 3 & 0 & 0 & 0 & 0 & 0 & 0 & 0 & 3 \\
\hline Gastropoda & Natica furva & 0 & 1 & 0 & 0 & 0 & 0 & 0 & 0 & 1 \\
\hline Gastropoda & Natica livida & 0 & 1 & 0 & 0 & 0 & 0 & 0 & 0 & 1 \\
\hline Gastropoda & Polynices lacteus & 0 & 0 & 0 & 0 & 1 & 0 & 0 & 0 & 1 \\
\hline Gastropoda & Raphitoma linearis & 0 & 0 & 0 & 1 & 0 & 0 & 0 & 0 & 1 \\
\hline Gastropoda & Smaragdia viridis & 4 & 0 & 0 & 0 & 0 & 0 & 0 & 0 & 4 \\
\hline Gastropoda & Turritella brocchii & 0 & 1 & 2 & 2 & 4 & 2 & 2 & 0 & 13 \\
\hline Gastropoda & Vexillum (Pusia) zebrinum & 0 & 0 & 1 & 0 & 0 & 0 & 0 & 0 & 1 \\
\hline & Total & 139 & 159 & 99 & 87 & 87 & 157 & 613 & 157 & 1498 \\
\hline
\end{tabular}

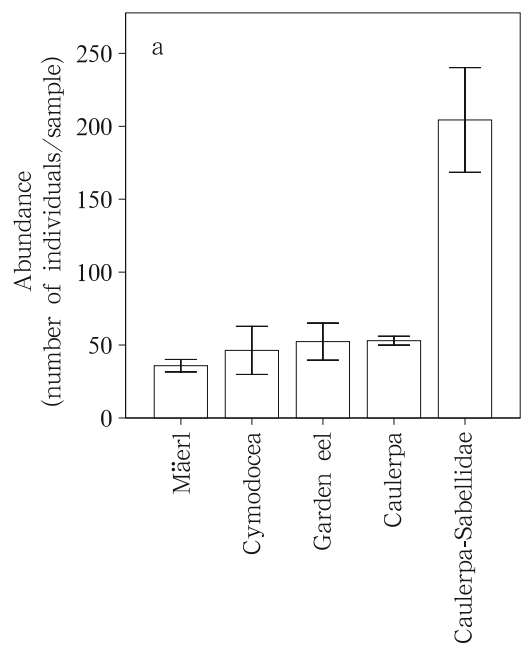

Seabed type

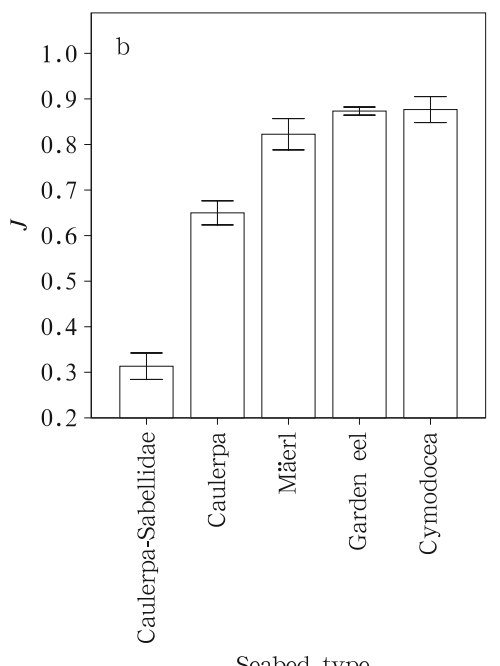

Seabed type

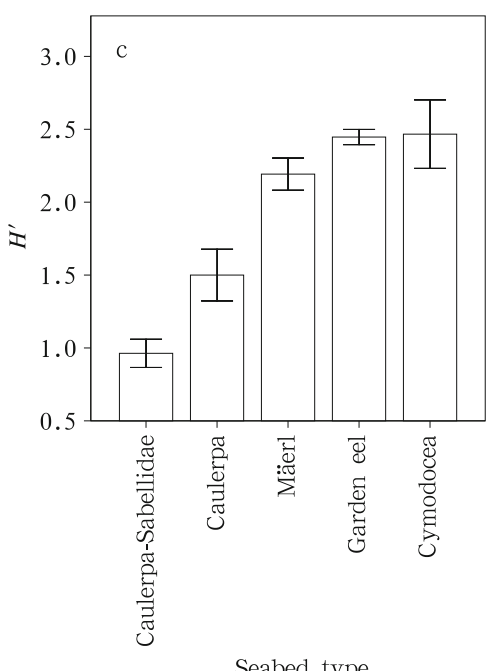

Seabed type

Fig.3. Patterns of variation in abundance and diversity measures for the studied seabeds. a. Relative abundance (number of individuals), b. evenness $(J)$, and c. Shannon's diversity $\left(H^{\prime}\right)$. Bars represent means \pm 1 S.E.

and Caulerpa meadows, with a mean value of 0.96 and 1.5, respectively (Fig. 3). Shannon's diversity presented significant differences among sedimentary habitats $(H=13.02 ; p=0.01)$.

\subsection{Multivariate analysis}

The eight sampling stations presented low overall similarity. Station GA-7 was separated at the $18.1 \%$ of similarity, being dominated by Bispira viola, naming the sabellid bottom of this sampling area. Station GA-2 was separated at $24.1 \%$ of similarity, with high abundances of the polychaetes Aponuphis bilineata and Demonax brachychona. Stations GA-1 and GA-8 were separated at the level of $29.7 \%$ of similarity, due to the presence of high densities of the tanaid Apseudes talpa and intermediate abundances of $A$. bilineata. The remaining sampling stations (GA-3, GA-
4, GA-5 and GA-6) presented a similarity of $38.6 \%$ and were characterized by the presence of the polychaetes A. bilineata and Chone filicauda, as well as the mollusc Turritella brocchii (Fig. 4).

Species richness was not significantly different amongst habitats (Table 4). The seabeds formed by Caulerpa with sabellids, however, presented significantly lower diversity and evenness, and higher macrofaunal abundance when compared with all the other habitat types, including Caulerpa meadows (Table 4). Caulerpa meadows were statistically similar in terms of abundance to the remaining habitats, but differed regarding diversity and evenness, which were by far lower than in the other habitats.

The organic matter content was inversely correlated with the polychaete Aponuphis bilineata, whilst the polychaete Ditrupa arietina showed an inverse cor- 


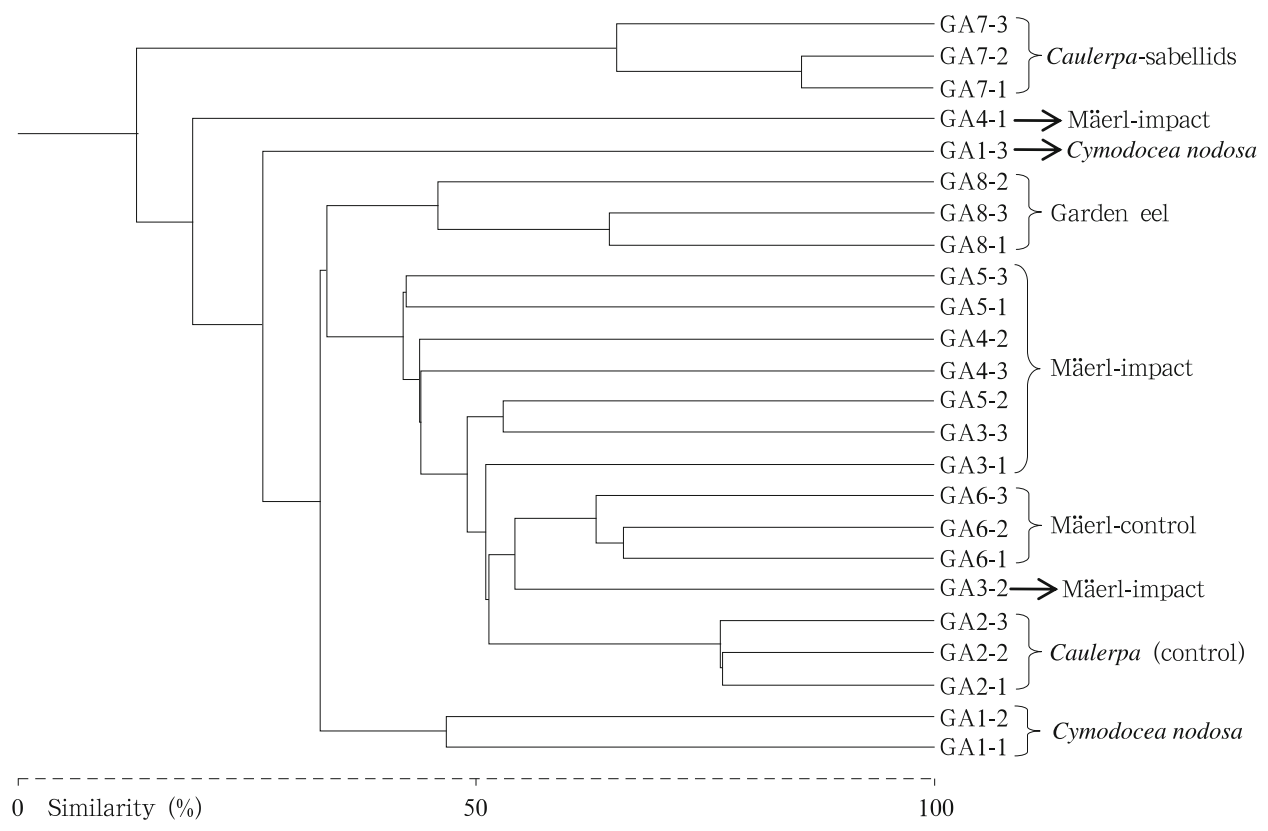

Fig.4. Bray-Curtis cluster analysis of sampling stations based on species composition and abundance per species.

Table 4. Comparison of mean abundance and diversity measures between seabed types by a two-sided Student's $t$ test (tests adjusted for pairwise comparisons with Bonferroni correction; $\alpha=0.05$ ). Capital letters indicate significant pairwise differences between habitats for each index [e.g., Evenness $(J)$ was significantly higher in Caulerpa bottoms than in habitats formed by Caulerpa plus sabellids]

\begin{tabular}{|c|c|c|c|c|c|}
\hline & \multicolumn{5}{|c|}{ Habitat } \\
\hline & Garden eel (A) & Caulerpa + sabellids (B) & Caulerpa $(\mathrm{C})$ & Maërl (D) & Cymodocea nodosa (E) \\
\hline Abundance ( $\mathrm{n}^{\circ}$ ind.) & & A C D E & & & \\
\hline Richness ( $\mathrm{n}^{\circ}$ spp.) & & & & & \\
\hline Evenness $(J)$ & B & & B & B & B \\
\hline Shannon's Diversity $\left(H^{\prime}\right)$ & B C & & & B & B C \\
\hline
\end{tabular}

Table 5. Correlations between the seven most abundant macrofaunal species and the most important abiotic factors ${ }^{1)}$

\begin{tabular}{|c|c|c|c|c|c|c|c|c|c|}
\hline Species & $\mathrm{OM}$ & $\mathrm{P}$ & Gravel & VCS & $\mathrm{CS}$ & MS & FS & VFS & Silt/clay \\
\hline Aponuphis bilineata & $-0.906^{* *}$ & & & & & & & & \\
\hline Ditrupa arietina & & $-0.936^{* *}$ & & & $0.849^{* *}$ & & & & \\
\hline Prionospio steenstrupii & $0.871^{* *}$ & & & & $0.830^{* *}$ & & & & \\
\hline Apseudes talpa & & & $-0.782^{*}$ & $-0.883^{* *}$ & $-0.934^{* *}$ & & $0.727^{* *}$ & $0.745^{*}$ & $0.744^{*}$ \\
\hline
\end{tabular}

relation with the content of phosphates and a positive correlation with coarse sands. The polychaete Prionospio steenstrupii presented a positive correlation with phosphates and medium sands. The tanaid Apseudes talpa showed the highest correlations with abiotic variables, and it was inversely correlated with coarser sedimentary fractions (gravels, very coarse sands and coarse sands) and positively correlated with the finer sedimentary types (fine sands, very fine sands and silt/clay) (Table 5).
As compared with sites in the Mediterranean Sea, Tenerife seabeds are characterized by low macrofaunal abundances and intermediate species diversity (Sciberras et al., 2009). This is mainly due to differences in grain size, since mäerl beds in the Mediterranean seabeds are characterized mainly by gravels $(>2$ $\mathrm{mm}$ diameter) that harbour an important number of crevicular spaces where inhabit a rich faunal community adapted to this environment (Barberá et al., 2003). Moreover, the Canarian archipelago is char- 
acterized to be an oligotrophic region (Barton et al., 1998), with low contents of particulate organic matter that implies the presence of low benthic abundances in all faunal components, mainly mega- $(>1 \mathrm{~mm})$ and macrofauna $(>0.5 \mathrm{~mm})$. Sedimentary habitats determined the macrofaunal community structure, with different granulometric fractions characterizing different habitats. There is a mosaic of seabed habitats determined mainly by granulometric variation, which enhances local habitat diversity without a large influence on taxonomic diversity. Cymodocea meadows are present in finer sediments whilst Caulerpa meadows and Maërl bottoms are on medium or coarse sands. In the habitat of Caulerpa and sabellids, Bispira viola clearly dominates the macrofaunal community structure, and the remaining species are comparatively scarcer. In Cymodocea meadows, Caulerpa meadows, mäerl seabeds and garden eel assemblages, the onuphid polychaete Aponuphis bilineata was dominant. This species was accompanied by the tanaid Apseudes talpa (Cymodocea nodosa meadows and Garden eel assemblages), the sabellid polychaete Demonax brachychona (Caulerpa meadows) and the sabellid polychaete Chone filicauda and the mollusc Turritella brochii (Mäerl seabeds).

This ecological study constitutes the first attempt to describe macrofaunal diversity in mäerl seabeds in the Canary islands. More detailed studies are necessary to understand ecosystem functioning of this fragile marine ecosystem. Moreover, this study can be used as a baseline for future conservation studies of mäerl seabeds that are subjected to anthropogenic stressors (pipelines, harbours, atificial beaches, spills, among others) or naturally-induced changes (e.g., climate change).

\section{Acknowledgements}

The authors acknowledge Humberto Aguirre for his help during sampling campaign. We are also grateful for all the hospitality provided by the company Parque Mar Paraso SL for financial support and also to the fishermen association from Las Galletas for logistic facilities.

\section{References}

Agnesi S, Annunziatellis A, Casese M L, et al. 2009. Analysis on the coralligenous assemblages in the Mediterranean Sea: a review of the current state of knowledge in support of future investigations. In: Pergent-Martini C, Brichet M, eds. UNEP-MAPRAC/SPA (2009) Proceedings of the 1st Mediterranean symposium on the conservation of the coralligenous and other calcareous bio-concretions (Tabarka, 15-16 January 2009). Tunis: RAC/SPA publication

Airoldi L, Beck M W. 2007. Loss, status and trends for coastal marine habitats of Europe. Oceanography and Marine Biological Annual Review, 45: 345-405

Ballesteros E. 2006. Mediterranean coralligenous assemblages: a synthesis of present knowledge. Oceanography and Marine Biology Annual Review, 44: 123195

Barberá C, Bordehore C, Borg J A, et al. 2003. Conservation and management of northeast Atlantic and Mediterranean maërl beds. Aquatic Conservation of Marine and Freswater Ecosystem, 13(S1): S65-S76

Barton E D, Arístegui J, Tett P, et al. 1998. The transition zone of the Canary Current upwelling region. Prog Ocean, 41: 455-504

Blake C, Maggs C A. 2003. Comparative growth rates and internal banding periodicity of maërl species (Corallinales, Rhodophyta) from northern Europe. Phycology, 42: 606-612

Buchanan J B, Kain M. 1971. Measurements of the physical and chemical environment. In: McIntyre A D, ed. Methods for the study of marine benthos. Oxford \& Edinburgh: Blackwell Scientific Publications, $30-58$

Clarke K R, Warwick R M. 2001. Changes in marine communities: an approach to statistical analysis and interpretation. Plymouth, UK: PRIMER-E

Kimberly A E, Roberts M G. 1905. A method for the direct determination of organic nitrogen by the Kjeldahl process. Public Health Pap Rep, 31(2): 109122

Rubino L, Catapano V, Guerra G. 1989. Determination of inorganic phosphorus in serum: Evaluation of three methods applied to the Technicon RA-1000 analyzer. Journal of Automatic Chemistry, 11(4): 164-167

Sciberras M, Rizzo M, Mifsud J R, et al. 2009. Habitat structure and biological characteristics of a maërl bed off the northeastern coast of the Maltese Islands (central Mediterranean). Marine Biodiversity, 39: 251-264

Walkley A, Black J A. 1934. An examination of the Degtjareff method for determining soil organic matter and a proposed modification of the chromic titration method. Soil Science, 37: 29-38 